\title{
REVIEW
}

\section{Medication adherence in inflammatory bowel disease}

\author{
Webber Chan ${ }^{1,2}$, Andy Chen ${ }^{3}$, Darren Tiao ${ }^{4}$, Christian Selinger ${ }^{5}$, Rupert Leong ${ }^{1}$ \\ ${ }^{1}$ Gastroenterology and Liver Services, Concord Repatriation General Hospital, Sydney, Australia, ${ }^{2}$ Department of Gastroenterology and \\ Hepatology, Singapore General Hospital, Singapore, ${ }^{3}$ South Western Sydney Clinical School, University of New South Wales, Sydney, ${ }^{4}$ Sydney \\ Medical School, the University of Sydney, Sydney, Australia, ${ }^{5}$ IBD Unit, Leeds Gastroenterology Institute, Leeds Teaching Hospitals NHS Trust, St \\ James University Hospital, Leeds, UK
}

Inflammatory bowel disease (IBD) is a chronic idiopathic inflammatory condition with intestinal and extraintestinal manifestations. Medications are the cornerstone of treatment of IBD. However, patients often adhere to medication poorly. Adherence to medications is defined as the process by which patients take their medications as prescribed. Treatment non-adherence is a common problem among chronic diseases, averaging $50 \%$ in developed countries and is even poorer in developing countries. In this review, we will examine the adherence data in IBD which vary greatly depending on the study population, route of administration, and methods of adherence measurement used. We will also discuss the adverse clinical outcomes related to non-adherence to medical treatment including increased disease activity, flares, loss of response to anti-tumor necrosis factor therapy, and so forth. There are many methods to measure medication adherence namely direct and indirect methods, each with their advantages and drawbacks. Finally, we will explore different intervention strategies to improve adherence to medications. (Intest Res 2017;15:434-445)

Key Words: Medication adherence; Colitis, ulcerative; Crohn disease; Inflammatory bowel disease

\section{INTRODUCTION}

Inflammatory bowel disease (IBD), comprising UC and $\mathrm{CD}$, a chronic idiopathic inflammatory condition possessing intestinal and extra-intestinal features and is characterized by periods of relapses and remission. Medications, including 5-aminosalicylates, corticosteroids, immunomodulators (thiopurines [azathioprine and mercaptopurine] and methotrexate), and biological agents (anti-tumor necrosis factor [TNF; infliximab, adalimumab, and certolizumab pegol], and anti-adhesion molecules [vedolizumab]), are the cornerstone of treatment of IBD. However, patient adherence to medication, like other chronic diseases, is often poor. The definition of medication adherence evolved over the past 4

Received May 17, 2017. Revised May 28, 2017.

Accepted May 29, 2017. Published online August 2, 2017

Correspondence to Webber Chan, Gastroenterology and Liver Services, Concord Repatriation General Hospital, ACE Unit, Level 1 West, Hospital

Road Concord NSW 2139, Australia. Tel: +61-2-9767-6111, Fax: +61-2-

9767-6767,E-mail: webber.chan.p.w@singhealth.com.sg

Financial support: None. Conflict of interest: None. decades. ${ }^{1-3}$ Sackett and Haynes ${ }^{4}$ were the first to introduce the term "compliance" to medicine in 1976. They defined "compliance" as the extent to which the patient's behavior (in terms of taking medications, following diets or executing other lifestyle changes) coincides with the clinical prescription. Since then various adaptations of the original definition took place, leading to misunderstanding and confusion, and hamper comparisons of results of medical research and application in practice. Hence, a new taxonomy for describing and defining adherence to medications has been proposed to promote consistency in terminology and methods so as to aid in the conduct, analysis and interpretation of scientific studies of medication adherence. ${ }^{5}$ Today the word "adherence" is preferred by many health care professionals, because "compliance," implies patients' passive obedience to the doctor's orders and that the treatment plan is not based on cooperation between physicians and patients.

\footnotetext{
๑ Copyright 2017. Korean Association for the Study of Intestinal Diseases. All rights reserved.

This is an Open Access article distributed under the terms of the Creative Commons Attribution Non-Commercial License (http://creativecommons.org/licenses/by-nc/4.0)

which permits unrestricted non-commercial use, distribution, and reproduction in any medium, provided the original work is properly cited.
} 


\section{DEFINITION OF ADHERENCE}

Adherence to medications is defined as the process by which patients take their medications as prescribed. ${ }^{5}$ It has 3 components: initiation (when the patient takes the first dose of a prescribed medication), implementation (the extent to which a patient's actual dosing corresponds to the prescribed dosing regimen, from initiation until the last dose is taken), and discontinuation (when the next dose to be taken is omitted and no more doses are taken thereafter). To achieve the full benefit of the many effective medications, patients have to follow prescribed treatment regimens reasonably closely. There is no consensus on the cutoff to define adequate adherence. Some clinical trials consider rates of greater than $80 \%$ to be acceptable, while others consider rates of greater than $95 \%$ to be an absolute requirement for adequate adherence.

Treatment non-adherence in chronic diseases averages $50 \%$ in developed countries and is even poorer in developing countries. ${ }^{2}$ In IBD, adherence data vary greatly according to study population (adults versus paediatric patients), route of administration, and methods of adherence measurement (e.g., blood analysis, pharmacy refill, self-report [diaries, interviews, and questionnaires]), and so forth. A systematic review $^{6}$ of 17 studies totalling 4,322 adult IBD subjects found non-adherence to oral medications ranging from $7 \%$ to $72 \%$. The reported medication non-adherence rate in Asian IBD patients ranged between $20 \%$ and $30 \%$. For example, Kawakami et al. ${ }^{7}$ found that $27.9 \%$ of their patients were nonadherent to aminosalicylate, whereas Kim et al. ${ }^{8}$ reported a $22.3 \%$ of non-adherence rate in their cohort of patients in Korea. Non-adherence to oral medications in adolescents with IBD has been found to range from $2 \%$ to $93 \% .{ }^{9}$ Jeganathan et al. ${ }^{10}$ recently observed that transition from paediatric to adult IBD service did not affect medication adherence. In that pilot study, non-adherence rates of young adults (age, 18-25 years), and paediatric patients (age, 12-18 years) were $17 \%$ and $5 \%$, respectively $(P=0.28)$. There were no significant differences in Medication Adherence Reporting Scale (MARS) scores between children, recently transitioned adults, other post-transitional adults, and never-transitioned adults. Adherence rate with biological therapy is higher. Selinger et al. ${ }^{11}$ (in abstract form only) reported an overall non-adherence rate of $30 \%$ to maintenance medication in an Australian cohort of IBD patients, with the highest adherent rate among patients on biological therapies (94.7\%). Lopez et al. ${ }^{12}$ performed a systematic review on the adherence rate to anti-TNF therapy in IBD. A total of 13 studies with 93,998 patients (both adult and paediatric patients) were included in the review. The pooled adherence rate to biologics in patients with IBD was $82.6 \%$, ranging from $36.8 \%$ to $96.0 \%$.

\section{EFFECTS OF NON-ADHERENCE}

Non-adherence in IBD is associated with an increase in disease activity, ${ }^{13}$ relapse, ${ }^{13,14}$ loss of response (LOR) to antiTNF agents, ${ }^{15}$ higher morbidity and mortality (e.g., with colorectal cancer), increased health expenditure, ${ }^{16}$ poor quality of life (QOL) $)^{17,18}$ and higher disability. ${ }^{19}$ Kane et al. ${ }^{13}$ followed a cohort of 99 consecutive patients who had UC in remission for more than 6 months and were on maintenance mesalamine. They found that those who were not adherent with medication had more than a 5-fold greater risk of recurrence than adherent patients. In a United States-based 10-year retrospective study of 13,062 patients with UC with a median follow-up of 6.1 years, low adherers to oral mesalazine had significantly increased the risk of flares in UC compared with high adherers (hazard ratio, $2.8 ; P<0.001$ ). ${ }^{14}$ Similarly, Robinson et al. ${ }^{20}$ observed from their retrospective study that non-adherence to mesalazine maintenance therapy was associated with significant increases in the risk of relapse. Interestingly, they also found that adherent patients who switched between mesalazine formulations had a 3.5fold greater risk of relapse than those who did not switch.

Non-adherence to anti-TNF therapy may result in immunogenicity and subsequent LOR to biological treatment. Few studies have examined the association between adherence to anti-TNF and LOR. van der Have et al. ${ }^{15}$ demonstrated that of those 128 IBD patients who were on either infliximab or adalimumab, adherence was negatively associated with LOR to anti-TNF.

The association between adherence to medication and healthcare costs in IBD has also been evaluated. Kane and Shaya ${ }^{16}$ reviewed a U.S. population-based insurance database and included patients who had a follow-up of more than 1 month and were prescribed at least one 5 -aminosalicylic acid (ASA) preparation. They demonstrated that adherence was associated with $62 \%$ lower costs for hospital admissions $(P<0.001), 13 \%$ lower for outpatient visits $(P<0.05)$, $45 \%$ lower for visits to the emergency department $(P<0.001)$, and $49.8 \%$ lower overall total health care costs compared with non-adherence.

Data on the association between drug adherence and health-related QOL (HRQOL) in IBD patients is conflicting. Hommel et al. ${ }^{17}$ studied the relationship between medication adherence and QOL in 36 adolescents with IBD. Medi- 
cation adherence was measured by the Medical Adherence Measure and pill counts. Paediatric QOL inventory was used to measure the adolescents' QOL. Non-adherence to 6-mercaptopurine (MP)/azathioprine was related to poorer patient-reported physical health QOL. In contrast, Horváth et al. ${ }^{18}$ enrolled 592 IBD patients to evaluate whether HRQOL influences medication adherence and vice versa. The patients completed the Short Form-36 (SF-36) questionnaire and a medication adherence report scale during their visits. It was concluded that there was no association between the sum of HRQOL and different subscores and non-adherence. Disability, which might more objectively measure the effects of IBD than QOL, was found to be significantly higher in non-adherers. ${ }^{19}$ Perry et al. ${ }^{19}$ did the first study to examine the relationship between non-adherence to medication and functional outcomes (using the IBD-disability index) in IBD patients. Disability was strongly correlated with medication non-adherence ( $\mathrm{r}=0.366$; median difference of IBD-disability index, $13.5 ; P<0.01)$.

\section{METHODS OF ADHERENCE MEASUREMENTS}

There are a number of ways of measuring drug adherence. The available methods for measuring adherence can be grouped into direct and indirect methods and no method is considered the gold standard. Direct measures include biochemical analysis such as checking drug metabolite levels in blood or urine sample and directly observed therapy (such as in tuberculosis treatment). Indirect methods include objective monitoring of medication usage (e.g., performing pill counts, pharmacy refills, and electronic monitoring devices) and subjective reports (e.g., patient-kept diaries, patient interviews, and self-report questionnaires) (Table 1).

\section{Drug Metabolites}

Thiopurines, including azathioprine or MP, have been widely used as steroid-sparing agents and are indicated to maintain remission in both UC and CD. Azathioprine is a prodrug that is rapidly cleaved to 6-MP in the liver. Subsequently, 6-MP is metabolized through a series of enzymatic pathways into 6-methylmercaptopurine (6-MMP) and the active metabolite, 6-thioguanine nucleotides (6-TGN). Several prospective studies have reported a significant correlation between 6 -TGN level of 235 to $450 \mathrm{pmol} / 8 \times 10^{8}$ red blood cell (RBC) and clinical response. ${ }^{21-23}$ Therapeutic drug monitoring (TDM) of thiopurine metabolites levels allows identification of non-adherent and refractory patients.

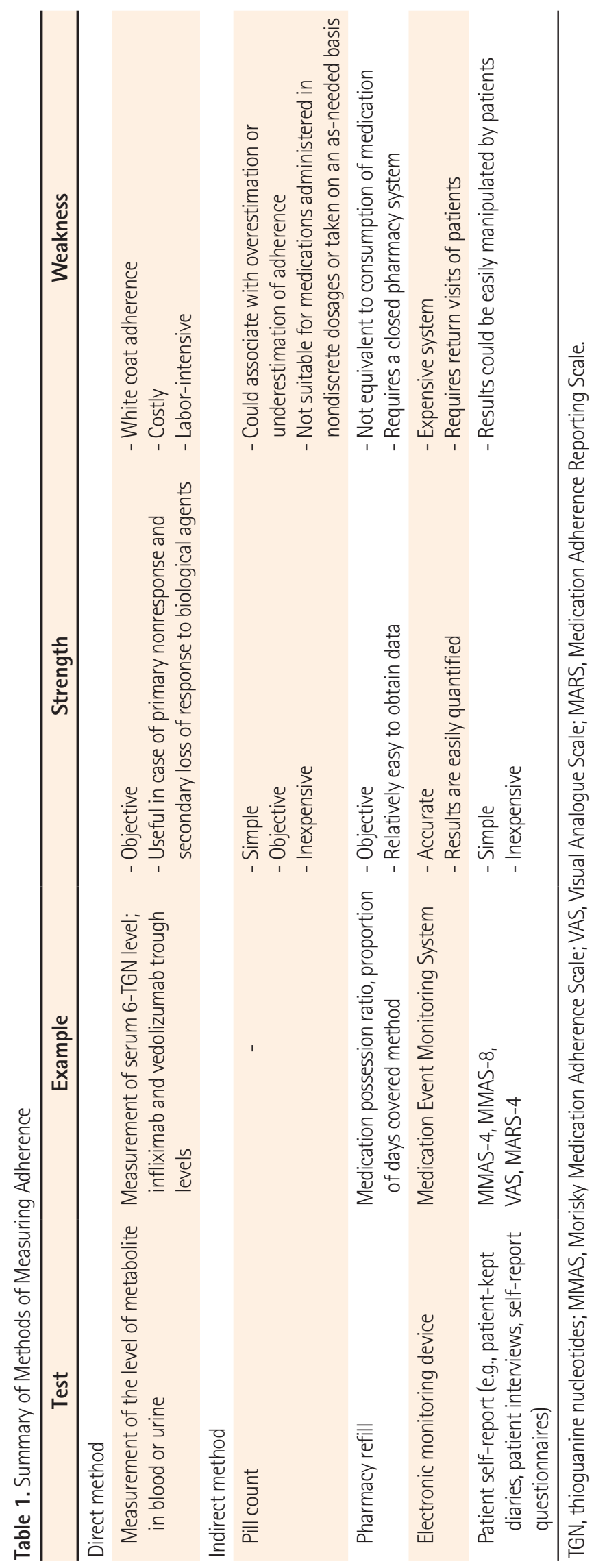


Thiopurine non-adherence was defined as 6-TGN levels $<100 \mathrm{pmol} / 8 \times 10^{8} \mathrm{RBC}$, in the absence of a metabolite profile suggesting hypermethylation of thiopurines to MMP (MMP, 6-TGN >11) when patients were adequately dosed..$^{24}$

TDM is also a promising tool to increase the efficacy, patient safety and cost-effectiveness of biological agents. It is useful in case of primary nonresponse and secondary LOR. While infliximab, a monoclonal antibody against TNF- $\alpha$, and vedolizumab, a monoclonal antibody against $\alpha_{4} \beta_{7}$ integrin, are administered at infusion centres (where adherence could be monitored), the SC anti-TNF (e.g., adalimumab and golimumab) are self-administered. TDM may have a role in monitoring adherence to these SC agents, but this indication has not been explored in clinical trials.

There are drawbacks associated with measuring drug metabolites. Variation in drug metabolism among individuals, and "white coat adherence," defined as "improved patient adherence to treatment around clinic visits," ${ }^{25}$ make an assessment of adherence difficult. Moreover, direct methods are costly and labour intensive to carry out. Finally, not all drug metabolite levels could be tested easily in the clinical setting. For methotrexate, there is limited and conflicting data on the usefulness of methotrexate metabolites as a measure of clinical response in IBD patients. ${ }^{26-28}$ With regards to mesalazine, although measurement of 5-ASA and n-acetyl-5-ASA, the metabolites of mesalazine, could be performed with gas or liquid chromatography-mass spectrometry (GC-MS or LCMS), ${ }^{29}$ they are costly and not widely available.

\section{Pill Counts}

Pill count of dosage units (e.g., capsules, tablets) that the patient has not taken by the scheduled clinic visit can be compared against the number of units received by the patient in the most recent prescription and the time since dispensing. Pill count adherence rate is calculated as the ([number of pills dispensed-number of pill returned]/number of pills prescribed) $\times 100 .^{30}$ This method is simple, objective and inexpensive but could associate with overestimation or underestimation of adherence, as when patients remove excess doses or refill medicines respectively before pill count is conducted. Moreover, pill counts are not suitable for medications administered in nondiscrete dosages or taken on an as-needed basis.

\section{Pharmacy Refill Data}

For pharmacy refill data to be valid, it is necessary that all patients obtain their medication from a centralized pharmacy in order to keep track of medicine refills. The major drawback of pharmacy refill records is their inability to determine if the patient actually takes the dispensed medication. At present, the 2 most commonly used methods for measuring medication adherence based on pharmacy data are the medication possession ratio (MPR; defined as the proportion of days' supply obtained over refill interval or fixed interval) and the proportion of days covered methods (PDC; defined as the number of days covered over a time interval). ${ }^{31}$ Patients with an MPR or PDC $\geq 80 \%$ are generally classified as adherent to their treatment.

\section{Electronic Monitoring Devices}

Electronic monitoring systems may provide a more reliable means of assessing adherence to both oral and topical medications. The Medication Event Monitoring System $\left(\right.$ MEMS $\left.^{\circledR}\right)$ medication bottles contain a microelectronic chip that registers the date and time of every bottle opening. Provided that each bottle openings represent medication intake, MEMS give a detailed profile of the patient's adherence behavior. Electronic monitoring is considered approximate a gold standard due to the high correlation between electronic estimates and clinical outcomes. However, it is expensive, and there is no guarantee that the medicine which is removed is actually consumed or administered correctly.

\section{Self-Reporting}

The most common method to measure medication adherence is the use of self-report measures. These methods include: (1) patient-kept diaries, (2) patient interviews, and (3) standardized, validated questionnaires. The advantages of these measures are that they are simple, easy, inexpensive, and have a high degree of specificity for non-adherence. However, they are subject to recall bias and the potential that participants give answers that suit the perceived expectations of their interviewer.

\section{1) Patient-Kept Diaries}

Diaries can track any prescribed behavior including medication consumption and diet and are supposed to be completed on a daily basis, soon after the medication or diet is taken. Studies on using patient diaries have confirmed their role as a reliable method for securing data. Unfortunately, diaries are cumbersome for patients to complete. Therefore, there are often significant missing data or the diaries are 
completed just prior to a clinic visit. Moreover, overestimation of compliance is common with patient diaries. Straka et al. $^{32}$ assessed the accuracy of patient-kept diaries relative to electronic monitoring of compliance. The study indicated that $67 \%$ of patients overestimated their compliance when using a self-recording tool. An average of $30 \%$ of diary entries was in error compared with the MEMS vial recordings.

\section{2) Patient Interviews}

Patient interview by physicians is an easy and inexpensive method to assess adherence. Interviewer's skill and the construction of the questions can affect the accuracy and validity of the interview. Open-ended, nonjudgmental questions are recommended; negative questions that seem to blame the patient for noncompliant will bias the answers given by patients. Examples that may encourage honest, open answers on medication adherence include: "People often have difficulty taking their medicine for 1 reason or another... Have you had any difficulty taking your medicine?," or "About how often would you say you miss taking your medicine?"

\section{3) Questionnaires}

There are a large number of self-report medication adherence questionnaires that are suitable for use in clinical or research settings. In a systematic review, Nguyen et al. ${ }^{33}$ identified 43 adherence scales that had been validated and compared against an objective measure of medication adherence. The identified adherence scales elicit information regarding different aspects of adherence including medication-taking behavior, barriers to and determinants of adherence and beliefs associated with adherence. Some of the commonly used questionnaires in IBD are described below.

(1) Four-item Morisky Medication Adherence Scale

The original 4-item self-reported Medication Taking Scale was developed by Morisky et al. ${ }^{34}$ to assesses the reasons why people might fail to adhere to antihypertensive medications-forgetting to take medicine, carelessness, and stopping the drug when feeling better or starting the drug when feeling worse. Despite fair psychometric properties (Cronbach's $\alpha$

Table 2. Four-Item Morisky Medication Adherence Scale

\begin{tabular}{l}
\hline \multicolumn{1}{c}{ Question } \\
\hline 1. Do you ever forget to take your (name of health condition) medicine? \\
2. Do you ever have problems remembering to take your (name of health condition) medication? \\
3. When you feel better, do you sometimes stop taking your (name of health condition) medicine? \\
4. Sometimes if you feel worse when you take your (name of health condition) medicine, do you stop taking it?
\end{tabular}

Table 3. Eight-Item Morisky Adherence Scale for IBD Patients

\begin{tabular}{|c|c|}
\hline Question & Scoring \\
\hline 1. Do you sometimes forget to take your IBD pills? & 1, No; 0 , yes \\
\hline $\begin{array}{l}\text { 2. People sometimes miss taking their medications for reasons other than forgetting. Thinking over the past } 2 \text { weeks, were there any } \\
\text { days when you did not take your medication? }\end{array}$ & 1, No; 0 , yes \\
\hline 3. Have you ever cut back or stopped taking your medication without telling your doctor because you felt worse when you took it? & 1, No; 0 , yes \\
\hline 4. When you travel or leave home, do you sometimes forget to bring along your IBD medication? & 1, No; 0 , yes \\
\hline 5. Did you take your IBD medicine yesterday? & 1, No; 0 , yes \\
\hline 6. When you feel like your IBD symptoms are under control, do you sometimes stop taking your medication? & 1, No; 0 , yes \\
\hline $\begin{array}{l}\text { 7. Taking medication everyday is a real inconvenience for some people. Do you ever feel hassled about sticking to your IBD } \\
\text { treatment plan? }\end{array}$ & 1, No; 0 , yes \\
\hline \multicolumn{2}{|l|}{ 8. How often do you have difficulty remembering to take your IBD medications? } \\
\hline Rarely/never & 1.00 \\
\hline Once in a while & 0.75 \\
\hline Sometimes & 0.50 \\
\hline Usually & 0.25 \\
\hline Always & 0 \\
\hline
\end{tabular}


reliability, 0.61 ), this scale has been used to measure medication adherence with asthma, hypertension, and other chronic diseases. The sensitivity and specificity of the scale were $81 \%$ and $44 \%$, respectively. The scale was later revised based on focus group discussions among patients being treated for active tuberculosis. The item asking: "Are you careless at times about taking your (name of health condition) medicine?" was replaced by a nonintentional, non-blaming item "Do you ever have problems remembering to take your (name of health condition) medication?" This version of the original scale became known as the 4-item Morisky Medication Adherence Scale (MMAS- 4$)^{35}$ which demonstrated high criterion validity and discriminant validity. ${ }^{36}$ Each of the 4 questions is given the dichotomous response of "yes" or "no" with the sum of "yes" answers providing a composite measure of non-adherence (Table 2). Patients score 1 point for every "yes" answer. A score of 0 indicates high adherence; a score of 1 or 2 indicates intermediate adherence; and a score of 3 or 4 indicates low adherence.

\section{(2) Eight-item Morisky Medication Adherence Scale}

In 2008, Morisky et al. ${ }^{37}$ supplemented the validated MMAS- 4 with additional items addressing the circumstances surrounding adherence behavior to develop the 8-item MMAS (MMAS-8). Each item measures a specific medication-taking behavior and not a determinant of adherence behavior. Response categories are dichotomous with yes/no for the first 7 items and a 5-point Likert response for the last item. The scale is scored by assigning a single point to each question answered "no" in questions 1-4 and 6-7. A point is given in question 5 for an answer of "yes." Question 8 is scored as per Table 3. As mentioned in Morisky's previous report, fewer than 6 points are interpreted as low adherence; 6-7 points as medium adherence; and 8 points as high adherence. The 8-item medication adherence scale has much better psychometric properties: Cronbach's $\alpha$ reliability was 0.83 ; using a cutpoint of $<6$, the sensitivity of the measure to identify patients with poor blood pressure control was estimated to be $93 \%$, and the specificity was $53 \%$. MMAS was subsequently validated with outstanding validity and reli- ability in patients with other chronic diseases. This was also the first adherence scale to be validated in IBD $^{38}$ although conflicting data exist on its performance in patients with IBD. In the validation study, Trindade et al. ${ }^{38}$ correlated continuous single-interval medication availability (CSA) and MPR to the MMAS- 8 scale. Of the 110 IBD patients in the study, MMAS-8 identified 54 patients as low adherers to their IBD medication and 56 patients as medium or high adherers. Eighty-five percent of low adherers had non-persistent fill rates, as per CSA, compared with $11 \%$ of medium or high adherers. In contrast, Kane et al. ${ }^{39}$ administered the MMAS-8 to 150 IBD patients. The median survey adherence score was 7. The MPR ranged from $0 \%$ (injectable biologic) to $75 \%$ (infliximab) by drug class. Only those on an immunomodulator had a survey score that positively correlated with adherence. Variation in study designs and inclusion criteria may have accounted for the differences in results from the 2 studies.

(3) Visual Analogue Scale

The Visual Analogue Scale (VAS) is a single item within the questionnaire, wherein subjects are asked, "What percentage of time do you take your daily prescribed medication?" The subjects are instructed to place an " $x$ " on a horizontal line that is marked by $0 \%$ and 100 in $10 \%$ intervals, with $100 \%$ indicating a perfect adherence (Fig. 1). Patients whose VAS is less than $80 \%$ are classified as poorly adherent, whereas patients with a VAS of $80 \%$ or greater are classified as highly adherent.

(4) Medication Adherence Reporting Scale-4

There are a few versions of the Medication adherence Reporting Scale (MARS) which include a 9-item, ${ }^{40} 5$-item and 4-item scale. ${ }^{41-43}$ The 4-item MARS is commonly used in IBD. ${ }^{42,43}$ The MARS measures adherence by assessing agreement with statements including "I alter the dose of these medicines," "I forget to take these medicines," "I decide to miss a dose of these medicines," and "I stop taking these medicines altogether" on 5-point Likert scales, ranging from always "1" to never "5." Scores for each of the 4 items are summed to give a total score ranging from 4 to 20 , with high-

Visual Analogue Scale

On the line below, please indicate by marking on the line which number corresponds to the

degree you consider how well you take your IBD medication. The far left means that you hardly

take your medication and the far right means that you are taking your medication very well (always).
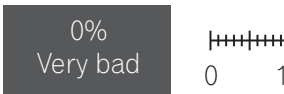

10

20

30

40

50

60

70

80

90100

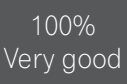

Fig. 1. Visual Analogue Scale. 
er scores indicating higher levels of adherence. Participants with scores between 4 and 16 are classified as low adherers, and those who score 17 to 20 are classified as high adherers.

In a recent study comparing 3 different tools to measure self-assessed medication adherence of patients with IBD, Severs et al. $^{44}$ found that the VAS most optimally represented the quantitative variability of adherence, whereas the MMAS-8 and the Forget Medicine Scale might have resulted in overestimation or underestimation of adherence due to unequal differences in outcome possibilities. It was concluded that VAS seems to be the most appropriate tool for quantifying medication adherence in clinical practice and that the MMAS-8 may be used additionally to provide insight into specific reasons for non-adherence.

\section{FACTORS ASSOCIATED WITH NON-ADHERENCE}

Identifying risk factors for non-adherence helps planning of intervention to improve adherence and clinical outcomes. In a recent systematic review, Jackson et al. ${ }^{6}$ acknowledged that existing literature had identified some significant associations between demographic, clinical, and psychosocial factors and non-adherence in IBD. However, heterogeneity in the inclusion criteria of articles may bias the results of systematic reviews. Based on their analysis, they found that none of the frequently measured demographic, clinical and treatment variables were consistently associated with non-adherence. Non-adherence to oral medication in IBD was more likely in younger patients, employed patients, unmarried patients, and those with shorter disease duration. Prescription of concomitant medications was generally associated with lower adherence. The relationship between psychological factors (depression and anxiety) and nonadherence remains to be determined. In Jackson et al's systematic review, psychological distress (depression, anxiety, psychiatric diagnosis, or chronic perceived stress), patients' beliefs about medications, and doctor-patient discordance were associated with non-adherence. In contrast, a later study by Selinger et al. ${ }^{43}$ showed that non-adherence in IBD was not associated with anxiety and depression. In addition, membership of an IBD patient organization was associated with better adherence.

In addition, recognizing that poor adherence to mesalamine is common, Moss et al. ${ }^{45}$ developed a 10-item survey to assesses UC patient-reported barriers to mesalamine adherence and predict those at risk for future non-adherence. They found that patients' beliefs about the need for maintenance mesalamine and their concerns about side effects influenced their adherence to mesalamine over time.

Recognizing that traditional ways of identifying and addressing non-adherence in IBD failed led to a paradigm shift in approaching the problem. Emerging concepts in the identification and prediction of non-adherence were the combination of "practicalities and perceptions approach" and the necessity-concerns framework. Horne et al. ${ }^{42}$ suggested that interventions to facilitate medication adherence would be more effective if they address both the practical factors (e.g., capacity and resources) influencing patients' ability to implement instructions to follow the agreed treatment plan and the perceptual factors (e.g., beliefs and preferences) influencing motivation to start and continue with treatment. The Necessity Concerns Framework ${ }^{46}$ assists clinicians to conceptualise and understand the key beliefs influencing adherence. It states that treatment adherence is associated with the way in which patients judge their personal need for a prescribed treatment relative to their concerns about its potential adverse effects. A further development to the Necessity Concerns Framework was the addition of attitudinal analysis based on patients' beliefs about medication using the Beliefs about Medicines Questionnaire. ${ }^{47}$ There are 4 attitude categories (Fig. 2): (1) accepting (high necessity, low concerns), (2) ambivalent (high necessity, high concerns), (3) skeptical (low necessity, high concerns), and (4) indifferent (low necessity, low concerns).

Horne et al. ${ }^{42}$ performed an attitudinal analysis on IBD patients and showed that compared to those who were "accepting" of maintenance therapies prescribed for IBD, participants in all 3 other attitudinal groups were significantly

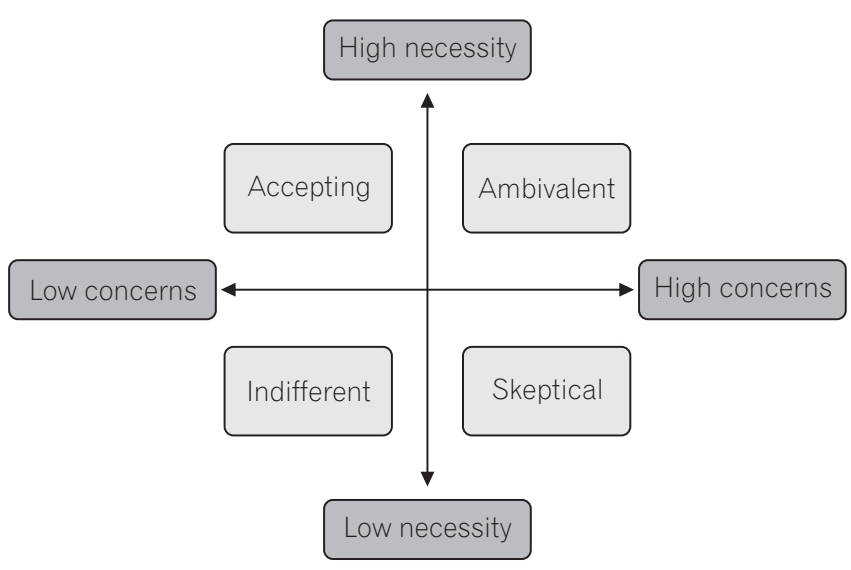

Fig. 2. Attitudinal analysis. Horne et al. ${ }^{42}$ showed that compared to those who were "accepting" of maintenance therapies prescribed for $I B D$, participants in all 3 other attitudinal groups were significantly more likely to be non-adherent. 
more likely to be non-adherent.

\section{INTERVENTIONS TO IMPROVE ADHERENCE}

There are various interventions to improve adherence in chronic diseases. However, the literature on interventions to enhance medication adherence in IBD is scarce and the reported studies varied widely with regards to patient population, intervention strategies, methods of adherence assessment, and clinical outcome measure, therefore making a comparison of results inappropriate. Moreover, they mainly concentrated on adult patients with UC and on promoting adherence to oral maintenance medications. They can be grouped into education, behavioral intervention, cognitivebehavioral intervention, and multifaceted approach.

\section{Education}

Educational interventions involve teaching patients about IBD aetiology, pathology, anatomy, investigations, medical and surgical treatment, mechanisms of action of the medication regimen, dosing schedule, potential side effects of treatment, consequences of non-adherence, disease course, nutrition, and complications of IBD. In the randomized controlled trial on effects of formal education for adult IBD patients, Waters et al. ${ }^{48}$ demonstrated a lower, though statistically nonsignificant, rate of medication non-adherence and health care use for the education group compared with standard care group. In another study, Tiao et al. ${ }^{49}$ (in abstract form only) investigated the effects and durability of IBD pharmacist targeted counselling intervention on adherence rates. Non-adherers were targeted for a structured personalized counselling session with an IBD pharmacist addressing misperceptions, concerns, risk and other queries. The study showed that targeted pharmacist counselling intervention on non-adherers effectively increased medication acceptance rates to be equivalent to adherers at 3 months and was durable to at least 15 months. Education however is rarely used alone in promoting medication adherence and always forms part of the multifaceted intervention.

\section{Behavioral Intervention}

Behavioral interventions are strategies designed to influence behavior through shaping, reminding (cues), or rewarding desired behavior (reinforcement) ${ }^{50}$ Such interventions include the use of visual or auditory reminder systems, use of weekly or daily pill box organisers (cues), dose simplification, and assessment of adherence with feedback to the patient (rewards and reinforcement), and so forth. Simplifying dosing schedule of mesalazine to once daily for maintenance of UC improves adherence. In a randomized pilot trial, Kane et al. ${ }^{51}$ randomized adults with UC to either once daily dosing (QD) or a conventional regimen (2 or 3 times daily dosing). At 3 months after initiation of the trial, all patients in the QD group were adherent, whereas only $70 \%$ of patients in the conventional dosing group were adherent. The adherence rate in the QD group remained significantly higher in the QD group at 6 months. In a subsequent multicenter, randomized, single blind, noninferiority trial, Dignass et al..$^{52}$ demonstrated that patients with UC given prolongedrelease oral mesalamine $2 \mathrm{~g}$ once daily had better remission rates, acceptability, and self-reported adherence to therapy (measured by VAS score) compared with those given oral mesalamine $1 \mathrm{~g}$ twice daily. Similarly, Suzuki et al..$^{53}$ also showed that once-daily dosing of a pH-dependent release mesalamine is as effective and safe as 3-times-daily dosing for maintenance of remission in Japanese patients with UC.

Use of audio-visual reminder systems (e.g., phone text reminder, pager text reminder, programmed electronic voice reminder device, etc.), and pill boxes are other practical ways to improve medication adherence. A meta-analysis of randomized controlled trials ${ }^{54}$ of reminder interventions revealed a statistically significant increase in adherence in groups receiving a reminder intervention compared to controls, whereas the type of reminder system did not seem to affect the adherence rate.

\section{Cognitive-Behavioral Therapy}

Cognitive behavioral interventions combine psychological and behavioral therapy aiming to promote adherence. Cognitive-Behavioral therapy (CBT) works on the principle that negative thoughts (e.g., not believing medications could alleviate symptoms) can block the abilities to act and learn new behaviors (e.g., consume medications at the prescribed time or refill medicines). CBT vary widely in content such as problem solving and increasing motivation to adhere. CBT alone has not been assessed in adult IBD patients. Problem solving skills training (PSST) has been evaluated as a standalone intervention to enhance adherence in paediatric IBD. Greenley et al. ${ }^{55}$ recruited 76 youth (age, $11-18$ years) on an oral IBD maintenance medication to participate in the study. Families randomized to a treatment group received either 2 or 4 PSST sessions. Modest increases in adherence occurred after 2 PSST sessions among those with imperfect baseline 
adherence and significant increases in adherence after 2 PSST sessions were documented for older adolescents.

\section{Multifaceted Approach}

In this approach, different strategies such as education, behavioral modification, CBT, motivational interviewing, telemedicine (with subtypes including telemonitoring, teleeducation, teleconsultation, and telecare), and so forth are employed in different combinations. The majority of the intervention studies to improve medication adherence in IBD used a multifaceted approach and proved useful in enhancing adherence in both adult and youth patients with IBD on oral treatment ${ }^{56-60}$ Motivational interviewing is a patientcentred counselling method used to elicit/strengthen motivation towards change.

Over the past 2 decades, advances in systems and information and communication technologies have influenced the practice of medicine. Telemedicine, simply defined as the application of information and communication technologies for providing health care services at a distance without the need for direct contact with the patient, ${ }^{61}$ is a promising tool in IBD management because they are safe and feasible applications with excellent patient acceptance. However, there are few robust data on many eHealth interventions outcome such as disease activity, medication adherence, cost-efficacy, and so forth, in comparison with the best available clinical care. In particular, the reported rates of adherence have been inconsistent between studies. Pedersen et al. ${ }^{62}$ performed a prospective, open-label, web-guided study with 3 months mesalazine therapy among patients with mild-to-moderate UC. Comparing week 0 with week 12 , a statistically significant difference in adherence by VAS $(P<0.001)$ and MARS $(P<0.001)$ was observed. In the randomized controlled trial of web-based patient management, Elkjaer et al. ${ }^{57}$ did not find statistically significant difference in adherence rates between the web and control groups at 12 months, although short-term adherence with acute treatment up to 4 weeks did improve significantly in the intervention group. In contrast, in another randomized, controlled trial of home telemanagement in patients with UC, the intervention did not improve disease activity, QOL, or adherence compared to best available care after 1 year. ${ }^{63}$ It seems therefore that webbased therapy can help to improve adherence in an acute flare of UC.

Telemedicine (telecare) has been used for adolescent patients with IBD. Hommel et al. ${ }^{64}$ developed an uncontrolled clinical pilot study to assess a telehealth behavioral

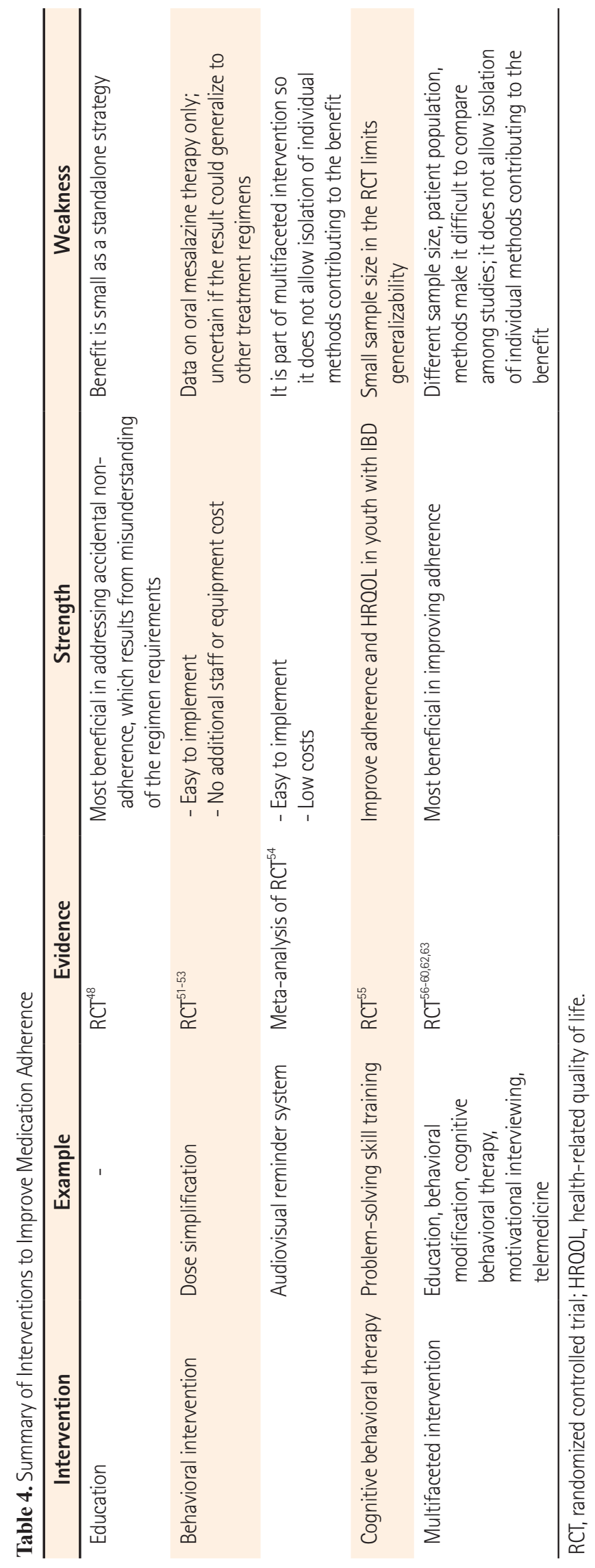


treatment for medication non-adherence in that group of patients. The program consisted of 4 weekly sessions, each lasting 60 to 90 minutes. The video sessions were conducted via Skype between patients and providers and psychologists. Although this program produced a slight decrease in adherence to thiopurine therapy from $61 \%$ at baseline to $53 \%$ after treatment (without different statistical differences due to the small sample size), it led to a meaningful increase in adherence to mesalamine therapy from $62 \%$ at baseline to $91 \%$. The investigators reported cost savings related to travel mileage and time.

Educational strategies, dose simplification, and use of audio-visual reminder systems have been proved in randomised clinical trials to be efficacious in improving medication adherence in IBD. They are most likely beneficial among patients who have accidental non-adherence due to misunderstanding of the treatment plan or complexity of the regimen. CBT is a promising intervention among paediatric IBD patients; more research is needed to test for its efficacy in adult IBD groups. There are more data on multifaceted interventions to improve adherence in IBD; the combined approach maximizes the potentials of each individual techniques to address different barriers to adherence such as motivational issues, problematic patterns of family functioning, and so forth. Identification of individual methods responsible for the improvement in compliance would aid in more resource been allocated to these strategies to benefit patients. Table 4 shows a summary of the various interventions.

\section{CONCLUSIONS}

Medication adherence is a significant problem in the management of IBD, which could lead to adverse clinical outcomes including an increase in disease activity, relapse, LOR to anti-TNF agents, higher morbidity and mortality, increased health expenditure, disability and possibly poor QOL. Various interventions exist, such as education, dose simplification, use of audio-visual reminder systems and multifaceted approach are proved efficacious to improve adherence. Telemedicine holds promise in the management of medication non-adherence, but more data from randomised controlled trials with larger sample size is needed to confirm its efficacy in comparison with the best available clinical care.

\section{ACKNOWLEDGEMENTS}

W.C. thanked Dr. Yunki Yau for proofreading the manuscript.

\section{AUTHOR CONTRIBUTION}

W.C. wrote the initial draft which was then extensively edited by A.C., D.T., C.S., and R.L. All authors approved the final draft. The manuscript, including related data, figures, and tables has not been previously published and that the manuscript is not under consideration elsewhere.

\section{REFERENCES}

1. Morisky DE. Facilitating treatment adherence: a practitioner's guidebook. Patient Educ Couns 1988;12:173-175.

2. Sabaté E. Adherence to long-term therapies: evidence for action. Geneva: World Health Organization, 2003.

3. Balkrishnan R. The importance of medication adherence in improving chronic-disease related outcomes: what we know and what we need to further know. Med Care 2005;43:517-520.

4. Sackett DL, Haynes RB. Compliance with therapeutic regimens. Baltimore: The Johns Hopkins University Press, 1976.

5. Vrijens B, De Geest S, Hughes DA, et al. A new taxonomy for describing and defining adherence to medications. Br J Clin Pharmacol 2012;73:691-705.

6. Jackson CA, Clatworthy J, Robinson A, Horne R. Factors associated with non-adherence to oral medication for inflammatory bowel disease: a systematic review. Am J Gastroenterol 2010;105:525-539.

7. Kawakami A, Tanaka M, Nishigaki M, et al. Relationship between non-adherence to aminosalicylate medication and the risk of clinical relapse among Japanese patients with ulcerative colitis in clinical remission: a prospective cohort study. J Gastroenterol 2013;48:1006-1015.

8. Kim SB, Kim KO, Jang BI, et al. Patients' beliefs and attitudes about their treatment for inflammatory bowel disease in Korea. J Gastroenterol Hepatol 2016;31:575-580.

9. Spekhorst LM, Hummel TZ, Benninga MA, van Rheenen PF, Kindermann A. Adherence to oral maintenance treatment in adolescents with inflammatory bowel disease. J Pediatr Gastroenterol Nutr 2016;62:264-270.

10. Jeganathan J, Lee $\mathrm{CH}$, Rahme A, et al. Medication adherence in transitional inflammatory bowel disease patients: a multicenter pilot study. J Gastroenterol Hepatol 2014;29:111.

11. Selinger C, Robinson A, Leong R. Non-adherence to inflammatory bowel disease maintenance medication: extent and predictors. J Gastroenterol Hepatol 2011;26:116.

12. Lopez A, Billioud V, Peyrin-Biroulet C, Peyrin-Biroulet L. Adherence to anti-TNF therapy in inflammatory bowel diseases: a systematic review. Inflamm Bowel Dis 2013;19:1528-1533. 
13. Kane S, Huo D, Aikens J, Hanauer S. Medication nonadherence and the outcomes of patients with quiescent ulcerative colitis. Am J Med 2003;114:39-43.

14. Khan N, Abbas AM, Bazzano LA, Koleva YN, Krousel-Wood M. Long-term oral mesalazine adherence and the risk of disease flare in ulcerative colitis: nationwide 10-year retrospective cohort from the veterans affairs healthcare system. Aliment Pharmacol Ther 2012;36:755-764.

15. van der Have M, Oldenburg B, Kaptein AA, et al. Non-adherence to anti-TNF therapy is associated with illness perceptions and clinical outcomes in outpatients with inflammatory bowel disease: results from a prospective multicentre study. J Crohns Colitis 2016;10:549-555.

16. Kane S, Shaya F. Medication non-adherence is associated with increased medical health care costs. Dig Dis Sci 2008;53:10201024.

17. Hommel KA, Davis CM, Baldassano RN. Medication adherence and quality of life in pediatric inflammatory bowel disease. J Pediatr Psychol 2008;33:867-874.

18. Horváth G, Farkas K, Hollósi R, et al. Is there any association between impaired health-related quality of life and non-adherence to medical therapy in inflammatory bowel disease? Scand J Gastroenterol 2012;47:1298-1303.

19. Perry J, Chen A, Collins G, Chang J, Kohler F, Leong R. Medication non-adherence in inflammatory bowel diseases is significantly associated with disability. J Gastroenterol Hepatol 2015;30(Suppl 3):135-136.

20. Robinson A, Hankins M, Wiseman G, Jones M. Maintaining stable symptom control in inflammatory bowel disease: a retrospective analysis of adherence, medication switches and the risk of relapse. Aliment Pharmacol Ther 2013;38:531-538.

21. Wright S, Sanders DS, Lobo AJ, Lennard L. Clinical significance of azathioprine active metabolite concentrations in inflammatory bowel disease. Gut 2004;53:1123-1128.

22. Cuffari C, Hunt S, Bayless T. Utilisation of erythrocyte 6-thioguanine metabolite levels to optimise azathioprine therapy in patients with inflammatory bowel disease. Gut 2001;48:642646.

23. Dubinsky MC, Lamothe S, Yang HY, et al. Pharmacogenomics and metabolite measurement for 6-mercaptopurine therapy in inflammatory bowel disease. Gastroenterology 2000;118:705713.

24. Haines ML, Ajlouni Y, Irving PM, et al. Clinical usefulness of therapeutic drug monitoring of thiopurines in patients with inadequately controlled inflammatory bowel disease. Inflamm Bowel Dis 2011;17:1301-1307.

25. Cramer JA, Scheyer RD, Mattson RH. Compliance declines between clinic visits. Arch Intern Med 1990;150:1509-1510.
26. Fong S, Ward MG, Nasr I, et al. PWE-111 higher red blood cell methotrexate polyglutamates correlate with increased disease activity, and are useful in assessing adherence. Gut 2014;63(Suppl 1):A173.

27. Brooks AJ, Begg EJ, Zhang M, Frampton CM, Barclay ML. Red blood cell methotrexate polyglutamate concentrations in inflammatory bowel disease. Ther Drug Monit 2007;29:619-625.

28. Egan LJ, Sandborn WJ, Tremaine WJ, et al. A randomized doseresponse and pharmacokinetic study of methotrexate for refractory inflammatory Crohn's disease and ulcerative colitis. Aliment Pharmacol Ther 1999;13:1597-1604.

29. Gifford AE, Berg AH, Lahiff C, Cheifetz AS, Horowitz G, Moss AC. A random urine test can identify patients at risk of mesalamine non-adherence: a prospective study. Am J Gastroenterol 2013;108:249-255.

30. Farmer KC. Methods for measuring and monitoring medication regimen adherence in clinical trials and clinical practice. Clin Ther 1999;21:1074-1090.

31. Andrade SE, Kahler KH, Frech F, Chan KA. Methods for evaluation of medication adherence and persistence using automated databases. Pharmacoepidemiol Drug Saf 2006;15:565-574.

32. Straka RJ, Fish JT, Benson SR, Suh JT. Patient self-reporting of compliance does not correspond with electronic monitoring: an evaluation using isosorbide dinitrate as a model drug. Pharmacotherapy 1997;17:126-132.

33. Nguyen TM, La Caze A, Cottrell N. What are validated selfreport adherence scales really measuring? A systematic review. Br J Clin Pharmacol 2014;77:427-445.

34. Morisky DE, Green LW, Levine DM. Concurrent and predictive validity of a self-reported measure of medication adherence. Med Care 1986;24:67-74.

35. Morisky DE, DiMatteo MR. Improving the measurement of selfreported medication nonadherence: final response. J Clin Epidemiol 2011;64:262-263.

36. Morisky DE, Malotte CK, Choi P, et al. A patient education program to improve adherence rates with antituberculosis drug regimens. Health Educ Q 1990;17:253-267.

37. Morisky DE, Ang A, Krousel-Wood M, Ward HJ. Predictive validity of a medication adherence measure in an outpatient setting. J Clin Hypertens (Greenwich) 2008;10:348-354.

38. Trindade AJ, Ehrlich A, Kornbluth A, Ullman TA. Are your patients taking their medicine? Validation of a new adherence scale in patients with inflammatory bowel disease and comparison with physician perception of adherence. Inflamm Bowel Dis 2011;17:599-604.

39. Kane S, Becker B, Harmsen WS, Kurian A, Morisky DE, Zinsmeister AR. Use of a screening tool to determine nonadherent behavior in inflammatory bowel disease. Am J Gastroenterol 2012;107:154-160. 
40. Horne R, Weinman J. Self-regulation and self-management in asthma: exploring the role of illness perceptions and treatment beliefs in explaining non-adherence to preventer medication. Psychol Health 2002;17:17-32.

41. Ediger JP, Walker JR, Graff L, et al. Predictors of medication adherence in inflammatory bowel disease. Am J Gastroenterol 2007;102:1417-1426.

42. Horne R, Parham R, Driscoll R, Robinson A. Patients' attitudes to medicines and adherence to maintenance treatment in inflammatory bowel disease. Inflamm Bowel Dis 2009;15:837844.

43. Selinger CP, Eaden J, Jones DB, et al. Modifiable factors associated with nonadherence to maintenance medication for inflammatory bowel disease. Inflamm Bowel Dis 2013;19:21992206.

44. Severs M, Zuithoff PN, Mangen MJ, et al. Assessing self-reported medication adherence in inflammatory bowel disease: a comparison of tools. Inflamm Bowel Dis 2016;22:2158-2164.

45. Moss AC, Lillis Y, Edwards George JB, et al. Attitudes to mesalamine questionnaire: a novel tool to predict mesalamine nonadherence in patients with IBD. Am J Gastroenterol 2014;109:1850-1855.

46. Horne R. Representations of medication and treatment: advances in theory and measurement. In: Petrie KJ, Weinman JA, eds. Perceptions of health and illness: current research and applications. London: Harwood Academic Press, 1997:155-187.

47. Horne R, Weinman J, Hankins M. The beliefs about medicines questionnaire: the development and evaluation of a new method for assessing the cognitive representation of medication. Psychol Health 1999;14:1-24.

48. Waters BM, Jensen L, Fedorak RN. Effects of formal education for patients with inflammatory bowel disease: a randomized controlled trial. Can J Gastroenterol 2005;19:235-244.

49. Tiao DK, Jeganathan J, Chen A. et al. Impact of targeted pharmacist counselling intervention on IBD medication adherence. J Gastroenterol Hepatol 2014;29:106.

50. Kripalani S, Yao X, Haynes RB. Interventions to enhance medication adherence in chronic medical conditions: a systematic review. Arch Intern Med 2007;167:540-550.

51. Kane S, Huo D, Magnanti K. A pilot feasibility study of once daily versus conventional dosing mesalamine for maintenance of ulcerative colitis. Clin Gastroenterol Hepatol 2003;1:170-173.

52. Dignass AU, Bokemeyer B, Adamek H, et al. Mesalamine once daily is more effective than twice daily in patients with quiescent ulcerative colitis. Clin Gastroenterol Hepatol 2009;7:762-769.
53. Suzuki Y, Iida M, Ito H, et al. $2.4 \mathrm{~g}$ Mesalamine (Asacol $400 \mathrm{mg}$ tablet) once daily is as effective as three times daily in maintenance of remission in ulcerative colitis: a randomized, noninferiority, multi-center trial. Inflamm Bowel Dis 2017;23:822-832.

54. Fenerty SD, West C, Davis SA, Kaplan SG, Feldman SR. The effect of reminder systems on patients' adherence to treatment. Patient Prefer Adherence 2012;6:127-135.

55. Greenley RN, Gumidyala AP, Nguyen E, et al. Can you teach a teen new tricks? Problem solving skills training improves oral medication adherence in pediatric patients with inflammatory bowel disease participating in a randomized trial. Inflamm Bowel Dis 2015;21:2649-2657.

56. Cook PF, Emiliozzi S, El-Hajj D, McCabe MM. Telephone nurse counseling for medication adherence in ulcerative colitis: a preliminary study. Patient Educ Couns 2010;81:182-186.

57. Elkjaer M, Shuhaibar M, Burisch J, et al. E-health empowers patients with ulcerative colitis: a randomised controlled trial of the web-guided 'Constant-care’ approach. Gut 2010;59:1652-1661.

58. Moshkovska T, Stone MA, Smith RM, Bankart J, Baker R, Mayberry JF. Impact of a tailored patient preference intervention in adherence to 5-aminosalicylic acid medication in ulcerative colitis: results from an exploratory randomized controlled trial. Inflamm Bowel Dis 2011;17:1874-1881.

59. Hommel KA, Herzer M, Ingerski LM, Hente E, Denson LA. Individually tailored treatment of medication nonadherence. J Pediatr Gastroenterol Nutr 2011;53:435-439.

60. Hommel KA, Hente EA, Odell S, et al. Evaluation of a groupbased behavioral intervention to promote adherence in adolescents with inflammatory bowel disease. Eur J Gastroenterol Hepatol 2012;24:64-69.

61. Aguas Peris M, Del Hoyo J, Bebia P, et al. Telemedicine in inflammatory bowel disease: opportunities and approaches. Inflamm Bowel Dis 2015;21:392-399.

62. Pedersen N, Thielsen P, Martinsen L, et al. eHealth: individualization of mesalazine treatment through a self-managed webbased solution in mild-to-moderate ulcerative colitis. Inflamm Bowel Dis 2014;20:2276-2285.

63. Cross RK, Cheevers N, Rustgi A, Langenberg P, Finkelstein J. Randomized, controlled trial of home telemanagement in patients with ulcerative colitis (UC HAT). Inflamm Bowel Dis 2012;18:1018-1025.

64. Hommel KA, Hente E, Herzer M, Ingerski LM, Denson LA. Telehealth behavioral treatment for medication nonadherence: a pilot and feasibility study. Eur J Gastroenterol Hepatol 2013;25:469-473. 